\title{
Linear Conjugacy in Biochemical Reaction Networks with Rational Reaction Rates
}

\author{
Attila Gábor • Katalin M. Hangos · Gábor \\ Szederkényi
}

Received: date / Accepted: date

\begin{abstract}
In this paper we show that the model form of a wide class of kinetic systems with rational terms in the reaction rates is invariant under a positive linear diagonal transformation. Thus, the concept of linear conjugacy defined originally for mass action systems is extended to rational biochemical models. The generalized Kirchhoff matrix and the kinetic weighting matrix of the linearly conjugate models are given as functions of the computed transformation parameters. It is shown through the illustrative examples that the dense realization of a linearly conjugate rational model may contain more reactions than that of a dynamically equivalent one due to the additional degrees of freedom introduced by the linear transformation. The proposed matrix-based representation is suitable for the computational search of preferred graph structures corresponding to linearly conjugate realizations of rational kinetic models.
\end{abstract}

Keywords chemical reaction networks · linear conjugacy · reaction graph · rational reaction rates

Attila Gábor

BioProcess Engineering Group, IIM-CSIC, Spanish Council for Scientific Research, Eduardo Cabello 6, 36208 Vigo, Spain

E-mail: attila.gabor@iim.csic.es

Katalin M. Hangos

Process Control Research Group, Institute for Computer Science and Control of the Hungarian Academy of Sciences (MTA SZTAKI), H-1111 Kende u. 13-17, Budapest, Hungary

Department of Electrical Engineering and Information Systems, University of Pannonia, H8200 Egyetem u. 10, Veszprém, Hungary

E-mail: hangos@scl.sztaki.hu

Gábor Szederkényi

Faculty of Information Technology and Bionics, Pázmány Péter Catholic University, H-1083 Prter u. 50/a, Budapest, Hungary

Process Control Research Group, Institute for Computer Science and Control of the Hungarian Academy of Sciences (MTA SZTAKI), H-1111 Kende u. 13-17, Budapest, Hungary

E-mail: szederkenyi@itk.ppke.hu 


\section{Introduction}

Kinetic systems obeying the mass action law are nonlinear polynomial models with a relatively simple algebraic structure but possessing advantageous dynamical descriptive properties [11]. Therefore, they are used not only to describe (bio)chemical processes but also non-negative systems from other application fields such as disease or population dynamics, and even transportation systems [5]. Moreover, it is possible to transform or embed originally non-kinetic systems into kinetic form [29,17]. Since the 1970's, the development of graph based analysis of dynamical systems [34], and of reaction network has been continuous, where an important aim is to characterize the qualitative dynamical behaviour of the network, preferably without precisely knowing its rate coefficients [18]. A few of the numerous important achievements in this area are the definition of deficiency and the corresponding Deficiency Zero and Deficiency One Theorems [13,14], a graph-theoretic approach for the analysis of stability and oscillations $[25,26]$, the consequences of injectivity properties [8], the notion and conditions of absolute concentration robustness [30], and recently, as a major breakthrough, the proof of the Global Attractor Conjecture for the one linkage class and possibly for the general case, respectively $[2,6]$.

One of the first mentioning of the possible non-uniqueness of the reaction graphs corresponding to a given kinetic dynamics is by Horn and Jackson [18]. This phenomenon is often called macro-equivalence, dynamical equivalence or confoundability [7]. The notion of dynamical equivalence was extended by introducing linear conjugacy by Johnston and Siegel allowing a linear diagonal state transformation between linearly conjugate networks [20]. It was shown that the computation of dynamically equivalent and linearly conjugate reaction graph structures having preferred properties such as (weak) reversibility, complex or detailed balance, minimal or zero deficiency corresponding to a set of kinetic ordinary differential equations can be solved efficiently in an appropriate optimization framework $[32,19,22,23]$.

The research field on the relation between the qualitative dynamics and the reaction graph structure of kinetic systems with non-mass-action kinetics has also showed considerable development recently. The increasing interest in this area is largely motivated by possible biological applications. Conditions for the persitence of chemical reaction networks with reaction rates satisfying monotonicity properties were given in [3] using the theory of Petri nets. The stability of a wide class of systems having general kinetics and strongly connected directed species-reaction (DSR) graphs was analyzed in [10]. New results on the injectivity and multistationarity of chemical reaction networks with general kinetics were given in [4]. The notion of generalized mass action (GMAK) systems was introduced in [27]. It was shown that deficiency zero weakly reversible GMAK systems with additional sign conditions have a unique positive steady state for arbitrary choice of rate constants and initial conditions. In [33] a realization procedure generalizing the result of [16] was given for kinetic models containing arbitrary reaction rates for constructing the so-called canonical reaction graph. In [15] the notion of dynamical equivalence was shown for systems with rational reaction rates using a representation based on the generalization of the Kirchhoff-matrix encoding the reaction graph structure. In the same paper, the computation of dense and sparse structures, i.e. network realizations containing the maximum and minimum possible number of reactions, 
respectively, was also described. These results clearly motivate the research of dynamical equivalence and the extension of previous graph structure computation methods for reaction network models with more general kinetics.

Based on the above results and motivating facts, the goal of this paper is to introduce linear conjugacy for rational kinetic systems and to show that the computation of linearly conjugate reaction graph structures can be handled in a computation framework that is similar to the one that was previously developed for mass action models [19].

\section{Background}

In this section, we briefly summarize the description of the studied class of reaction networks based on [15], where more details can be found.

We will denote the positive and non-negative orthant of the $n$-dimensional coordinates system with $\mathbb{R}_{+}^{n}$ and $\overline{\mathbb{R}}_{+}^{n}$, respectively. For any $c \in \mathbb{R}^{n}, \operatorname{diag}(c)$ denotes an $n \times n$ diagonal matrix, where the $i$-th diagonal entry is $c_{i}$. Moreover, the set of non-negative integers is denoted by $\mathbb{N}_{0}$.

\subsection{Basic components of reaction networks}

We consider closed thermodynamic systems under ideal conditions (isobar and isotherm), where chemical species $\mathrm{X}_{1}, \mathrm{X}_{2}, \ldots \mathrm{X}_{n}$ take part in chemical reactions of the form

$$
\sum_{k=1}^{n} \nu_{k i} \mathrm{X}_{k} \stackrel{r_{i j l}}{\longrightarrow} \sum_{k=1}^{n} \nu_{k j} \mathrm{X}_{k}
$$

The notations used in Eq. (1) are the following. The indices of the source and product complexes are denoted by $i$ and $j$, respectively. The stoichiometric coefficients of the reactants are denoted by $\nu_{1 i}, \ldots, \nu_{n i}$ and that of the products by $\nu_{1 j}, \ldots, \nu_{n j}$. The concentrations of the species $X_{i}$ are denoted by $x_{i}$ for $i=1, \ldots n$, and these are the coordinates of the non-negative state vector $x$. The non-negative integer linear combinations of the species $C_{i}=\sum_{k=1}^{n} \nu_{k i} \mathrm{X}_{k}$ and $C_{j}=\sum_{k=1}^{n} \nu_{k j} \mathrm{X}_{k}$ in (1) are called the complexes and are denoted by $C_{1}, \ldots C_{m}$. The coefficients of the complexes are stored in the complex composition matrix $Y \in \mathbb{R}^{n \times m}$ such that $[Y]_{i j}=\nu_{i j}$, i.e. the $i$-th column of the matrix stores the stoichiometric coefficients in the complex $C_{i}$.

The reaction rate function $r_{i j k}: \overline{\mathbb{R}}_{+}^{n} \mapsto \overline{\mathbb{R}}_{+}$describes how much product is generated in a unit time and it is often a non-linear function of the concentration vector. The indices $i, j$, and $k$ correspond to the source complex, product complex, and kinetics, respectively. In general, it is possible that the species in a complex give rise to multiple reaction paths, thus multiple different reaction rate functions can be assigned to each complex. These reaction rates are categorized based on their mathematical formulations. Using the notations of complexes and kinetics, Eq. (1) can be equivalently written as $C_{i} \stackrel{k_{i j l}, g_{i l}}{\longrightarrow} C_{j}$, where the reaction rate $r_{i j l}$ of the reaction is decomposed as

$$
r_{i j l}(x)=k_{i j l} \cdot g_{i l}(x)
$$


where $k_{i j l} \in \mathbb{R}_{+}$is a constant, non-negative principal reaction rate coefficient and $g_{i l}$ is a function of the species concentrations. Assuming the law of mass action the form of the kinetics is $g_{i l}(x)=\prod_{k=1}^{n} x^{\nu_{k i}}$. However, in biological applications the reaction rates are often not limited to mass action kinetics (MAK), but we assume that they can be sorted to a finite set of biochemical kinetics $\mathcal{G}_{i}=\left\{G_{1}, G_{2}, \ldots G_{d_{i}}\right\}$ in the case of each complex $C_{i}$. Here $d_{i}$ is the cardinality of $\mathcal{G}_{i}$, for $i=1 \ldots m$. Each of these kinetics defines a relationship among the species of the complex, for example $G_{1}=$ 'Mass action kinetics', $G_{2}=$ 'Michelis Menten kinetics', $G_{3}=$ 'Hill kinetics' etc. With this notation $g_{i l}(x)$ is associated with the kinetics $G_{l}$ of the complex $C_{i}$.

In most kinetic biochemical ODE models the reaction rate functions have polynomial or rational function form. Thus, from now on we assume that the reaction rate can be written as a ratio of two terms as

$$
g_{i l}(x)=\frac{\Psi_{i}(x)}{D_{i l}(x)}
$$

where $\Psi_{i}(x)$ is a monomial function $\left(\Psi_{i}(x)=\prod_{k} x_{k}^{\nu_{k i}}\right)$ and $D_{i l}(x)$ is a positive polynomial function of the concentration vector, i.e. it has non-negative coefficients and a positive zero-order term. To make the decomposition (2) unique, $g_{i l}(x)$ must not contain any linear scaling constant and thus we fix the zeroorder term in the denominator polynomial to 1 , i.e. $D_{i l}(x)$ is written as $D_{i l}(x)=$ $1+\sum \alpha_{m_{1}, m_{2}, \ldots m_{n}} x_{1}^{m_{1}} x_{2}^{m_{2}} \ldots x_{n}^{m_{n}}$ where $\alpha \in \mathbb{R}_{0,+}$ and $m_{1}, m_{2}, \ldots, m_{n}$ are nonnegative integers.

Since many of the kinetic biochemical ODE models can be represented in this form, we call these models biochemical reaction networks, or shortly, bio-CRNs [15]. Then we can characterize the bio-CRNs with the following four sets:

1. $\mathcal{S}=\left\{\mathrm{X}_{1}, \ldots \mathrm{X}_{n}\right\}$ is the set of species or chemical substances.

2. $\mathcal{C}=\left\{C_{1} \ldots C_{m}\right\}$ is the set of complexes.

3. $\mathcal{G}=\cup_{i=1}^{m} \mathcal{G}_{i}$ the set of reaction rates (kinetics).

4. The set of biochemical reactions is

$$
\begin{array}{r}
\mathcal{R}=\left\{\left(C_{i}, C_{j}, G_{l}\right) \mid C_{i}, C_{j} \in \mathcal{C}, G_{l} \in \mathcal{G}_{i} \text { and } C_{i}\right. \text { is transformed to } \\
\left.C_{j} \text { with the kinetics } G_{l}\right\} .
\end{array}
$$

The set of species, complexes and reactions with the kinetics uniquely determines the biochemical reaction network which is denoted by $\Sigma=(\mathcal{S}, \mathcal{C}, \mathcal{G}, \mathcal{R})$.

\subsection{Reaction graph}

The set of complexes together with the set of reactions give rise to the following directed, weighted graph representation of the bio-CRNs. The reaction graph $D=$ $\left(V_{d} ; E_{d}\right)$ consists of a finite non-empty set $V_{d}$ of vertices (nodes), which represent the complexes $V_{d}=\left\{C_{1}, \ldots C_{m}\right\}$, and a finite set of directed edges $E_{d}$, which represent the reactions. The edges are defined by triplets of the form $e_{(i, j, l)}=$ $\left(C_{i}, C_{j}, G_{l}\right)$ for $i, j=1, \ldots, m, i \neq j, l=1, \ldots, d_{i}$, where $i, j$ and $l$ are the indices of the source complex, product complex and the kinetics of the reaction, respectively. Furthermore, the principal reaction rate coefficients and the reaction kinetics are also used as weights on the edges. 
Note that the introduced description allows multiple directed edges with different kinetics converting $C_{i}$ to $C_{j}$ for any $i, j=1, \ldots, m, i \neq j$, which is a significant difference from the reaction graph of mass action systems, where multi-edges are naturally not allowed. However, similarly to mass action CRNs, bio-CRNs also cannot contain loop-edges.

\subsection{Dynamical equations of bio-CRNs}

In bio-CRNs the reactions are consuming species of the source complexes while producing species in the product complexes. The corresponding equations give rise to a dynamic ODE model which describes the concentration trajectories of the species. These models have a special structure, which allows us to read out the structure of the reaction graph from the equations. Motivated by the ODE structure of CRNs with MAKs [13], the dynamics of the bio-CRN is written in the following form

$$
\dot{x}=Y \cdot A_{k} \cdot P(x) \cdot \Psi(x),
$$

where $x \in \overline{\mathbb{R}}_{+}^{n}$ is the concentration vector of the species, $Y \in \mathbb{N}_{0}^{n \times m}$ is the complex composition matrix and $\Psi: \overline{\mathbb{R}}_{+}^{n} \rightarrow \overline{\mathbb{R}}_{+}^{m}$ is a vector function such that each element is a monomial

$$
\Psi_{i}(x)=\prod_{j=1}^{n} x_{j}^{Y_{j i}}, \quad i=1, \ldots, m
$$

The generalized Kirchhoff matrix $A_{k} \in \mathbb{R}^{m \times \kappa}$ stores the principal reaction rate coefficients and it is a matrix with zero column sums. When only one kinetics is associated to each complex, for example in mass action networks, $A_{k}$ is a square matrix, such that $\left[A_{k}\right]_{i j}=k_{j i}(i \neq j)$ is the reaction rate coefficient of the reaction from complex $C_{j}$ to complex $C_{i}$, and each diagonal element is the negative columnsum of corresponding off-diagonal elements, i.e. $\left[A_{k}\right]_{i i}=-\sum_{j=1, j \neq i}^{m}\left[A_{k}\right]_{j i}$.

In the general bio-CRN case, maybe more than one kinetics is associated to each complex. In this case let us denote the number of kinetics corresponding to complex $C_{i}$ by $d_{i}$. Then $A_{k} \in \mathbb{R}^{m \times \kappa}$ (where the total number of kinetics is denoted by $\left.\kappa=\sum_{i=1}^{m} d_{i}\right)$ can be written as a block matrix composed of $m$ blocks of size $m \times d_{i}$ as

$$
A_{k}=\left[A_{k}^{(1)} \ldots A_{k}^{(i)} \ldots A_{k}^{(m)}\right] .
$$

The $j$-th row of the block $A_{k}^{(i)}(i \neq j)$ contains the $d_{i}$ principal reaction rate coefficients of the reactions from the complex $C_{i}$ to complex $C_{j}$ with kinetics index $l \in\left\{1, \ldots, d_{i}\right\}$ i.e., $\left[A_{k}^{(i)}\right]_{j l}=k_{i j l}$. Furthermore, the elements of the $i$-th row contain the negative sum of the other column elements of the same column as follows: $\left[A_{k}^{(i)}\right]_{i l}=-\sum_{j=1, j \neq i}^{m}\left[A_{k}^{(i)}\right]_{j l}$. Thus, $A_{k}$ is a column conservation matrix (the sum of each column is 0 ). Naturally, $\left[A_{k}^{(i)}\right]_{j l}=0$ means that there is no reaction in the network from complex $C_{i}$ to $C_{j}$ with kinetics index $l$. Due to this construction, the generalized Kirchhoff matrix has a close relationship with the graph of the reaction network: the non-zero elements correspond to the edges of the reaction graph and the location of the non-zero elements together with the numerical values defines the weights of the edges of the graph. The locations of the non-zero elements will be referred to as the structure of the matrix $A_{k}$. 
The kinetic weighting mapping $P: \mathbb{R}^{n} \rightarrow \mathbb{R}^{\kappa \times m}$ arranges the denominator terms of the reaction rate functions (c.f. (2)) into a matrix form as follows

$$
P(x)=\left[\begin{array}{cccc}
P^{(1)}(x) & 0 & \ldots & 0 \\
0 & P^{(2)}(x) & \ldots & 0 \\
& \vdots & & \\
0 & 0 & \ldots & P^{(m)}(x)
\end{array}\right] .
$$

Here each block $P^{(i)}$ (for $i=1 \ldots m$ ) is of size $d_{i} \times 1$ and contains the denominators of the kinetics $g_{i 1}, g_{i 2}, \ldots g_{i d_{i}}$, for example $\left[P^{(i)}\right]_{l}=\frac{1}{D_{i l}(x)}$. The value of $P$ at any $x$ will be called a kinetic weighting matrix. Note that for MAL-CRNs the matrix $P(x)$ is the $m$-dimensional identity matrix.

From the above, it is clear that the bio-CRN $\Sigma$ can be equivalently characterized either by $\Sigma=(\mathcal{S}, \mathcal{C}, \mathcal{G}, \mathcal{R})$ or by the set $\Sigma=\left(Y, A_{k}, P\right)$. While the former can primarily be used for the analysis of the network, the latter is more suitable for computational purposes.

\subsection{Network realization and dynamical equivalence}

Given a set of ordinary differential equations, the realization problem is to find a possible bio-CRN with dynamics that is identical to the given equations. When the ODEs are given in the form

$$
\dot{x}=M g(x),
$$

where $M \in \mathbb{R}^{n \times \kappa}$ is a coefficient matrix and $g: \mathbb{R}^{n} \rightarrow \mathbb{R}^{\kappa}$ is a kinetic vector function with each of its elements being in the rational form (3). Gábor et al in [15] provided necessary and sufficient conditions for the existence of such a network $\Sigma=\left(Y, A_{\mathrm{k}}, P\right)$. If these conditions are fulfilled, we can write the right hand side of $(8)$ as

$$
M g(x)=Y A_{\mathrm{k}} P(x) \Psi(x) \text { for all } x \in \mathbb{R}_{+}^{n},
$$

where $M=Y \cdot A_{k}$ and $g(x)=P(x) \Psi(x)$. Moreover, $Y, A_{\mathrm{k}}, P$, and $\Psi$ have the properties described in subsection 2.3. If (9) holds, then $\Sigma=\left(Y, A_{k}, P\right)$ is called a dynamically equivalent realization of the kinetic system (8).

Similarly to the mass action case, it is easy to show that the realization problem does not have a unique solution in general, but one can find many possible different structures (simple examples can be found in [15]). Therefore, we say that the bioCRNs $\Sigma^{\prime}=\left(Y^{\prime}, A_{\mathrm{k}}^{\prime}, P^{\prime}\right)$ and $\Sigma^{\prime \prime}=\left(Y^{\prime \prime}, A_{\mathrm{k}}^{\prime \prime}, P^{\prime \prime}\right)$ are dynamically equivalent, if they give rise to the same dynamic equations of the form (8), i.e.

$$
M g(x)=Y^{\prime} A_{\mathrm{k}}^{\prime} P^{\prime}(x) \Psi^{\prime}(x)=Y^{\prime \prime} A_{\mathrm{k}}^{\prime \prime} P^{\prime \prime}(x) \Psi^{\prime \prime}(x) \quad \text { for } \forall x \in \overline{\mathbb{R}}_{+}^{n},
$$

where $Y^{\prime}, Y^{\prime \prime}$ are non-negative integer type matrices, $A_{\mathrm{k}}^{\prime}, A_{\mathrm{k}}^{\prime \prime}$ are generalized Kirchhoff matrices, $P^{\prime}, P^{\prime \prime}$ are rate weighting functions and $\Psi^{\prime}, \Psi^{\prime \prime}$ are computed from $Y^{\prime}$ and $Y^{\prime \prime}$, respectively, according to (5).

Many structural (graph) properties of the network are realization dependent, thus it is of significant interest to find dynamically equivalent realizations with given properties (for example reversible, complex balanced or weakly reversible realization) as formulated in [15]. 


\section{Linearly conjugate biochemical reaction networks}

Johnston and Siegel [20] introduced the notion of linearly conjugate networks for mass action systems. Two networks are said to be linearly conjugate if the solutions of their dynamic equations can be transformed into each other by a positive linear transformation. In general, the kinetic property of polynomial models (forming a subset of bioCRNs studied in this paper) is preserved at most under the positive scaling and/or re-ordering of the state variables $[12,20]$. It is easy to see that the original and linearly transformed networks share qualitative dynamical properties. Thus, if there is a network with known properties, for example the known number of equilibrium points and the stability properties of these equilibria, these properties are naturally transferred to all of its linearly conjugate networks.

The structure of biochemical reaction networks summarized in the previous section let us extend the linear conjugacy concept to bio-CRNs, i.e. to a wide class of non-mass-action kinetic systems.

We need the following three short lemmas to proceed to the linear conjugate biochemical reaction networks. The first lemma considers the positive linear scaling of a generalized Kirchhoff matrix. The second and third lemmas show results about the behaviour of the kinetic weighting matrix under the considered linear diagonal transformation, and in the case of a multiplication by a diagonal matrix from the right, respectively.

Lemma 1 Let $A_{k} \in \mathbb{R}^{m \times \kappa}$ be a generalized Kirchhoff matrix corresponding to a network of $m$ complexes and $d_{1}, d_{2} \ldots d_{m}$ kinetics $\left(\kappa=\sum_{i=1}^{m} d_{i}\right)$ which belong to the complexes. Further on, let $H$ be a positive diagonal matrix $H=\operatorname{diag}(c)$, where $c \in \mathbb{R}_{+}^{\kappa}$. Then, $A_{k}^{\prime}=A_{k} \cdot H$ is also a generalized Kirchhoff matrix with the same structure (same locations of the non-zero elements) as $A_{k}$.

Proof The $i$-th column of $A_{k}$ is multiplied by $c_{i}$, which do not alter the location of the zero and non-zero elements of the column and the column-sum remains zero.

Since the generalized Kirchhoff matrix has a strong relationship to the reaction graph, this means that, a linear scaling can change the weights of the directed edges, but not the structure of the reaction graph. The following lemma considers the linear transformation of the variables in the kinetic weighting function $P$ as defined in (7).

Lemma 2 Let $P: \mathbb{R}^{n} \rightarrow \mathbb{R}^{\kappa \times m}$ be a kinetic weighting mapping corresponding to a network of $m$ complexes and $d_{1}, d_{2} \ldots d_{m}$ kinetics $\left(\kappa=\sum_{i=1}^{m} d_{i}\right)$ corresponding to the complexes. Furthermore, let $T$ be a positive diagonal matrix $T=\operatorname{diag}(c)$, where $c \in \mathbb{R}_{+}^{n}$. Then $\hat{P}(x)=P(T x)$ is also a kinetic weighting mapping with the same structure as $P$.

Proof The invariance of the structure (the position of the non-zero entries) under a linear scaling is straightforward from the construction of $P$, see (7). However, the coefficients of the polynomials in the denominators will change. Recall that, the $l$-th element of the $i$-th block in $(7)$ is $\left[P^{(i)}\right]_{l}(x)=\frac{1}{D_{i l}(x)}$, where $D_{i l}(x)$ is a polynomial with positive coefficients $\left(\alpha_{m_{1}, m_{2}, \ldots m_{n}}>0\right)$ and constant term equal 
to 1 , i.e. $D_{i l}(x)=1+\sum \alpha_{m_{1}, m_{2}, \ldots m_{n}} x_{1}^{m_{1}} x_{2}^{m_{2}} \ldots x_{n}^{m_{n}}$. The linear scaling alters the elements as

$$
\begin{aligned}
D_{i l}(T x) & =1+\sum \alpha_{m_{1}, m_{2}, \ldots m_{n}} c_{1}^{m_{1}} x_{1}^{m_{1}} c_{2}^{m_{2}} x_{2}^{m_{2}} \ldots c_{n}^{m_{n}} x_{n}^{m_{n}} \\
& =1+\sum \tilde{\alpha}_{m_{1}, m_{2}, \ldots m_{n}} x_{1}^{m_{1}} x_{2}^{m_{2}} \ldots x_{n}^{m_{n}} \\
& =\tilde{D}_{i l}(x)
\end{aligned}
$$

where the new coefficients are $\tilde{\alpha}_{m_{1}, m_{2}, \ldots m_{n}}=\alpha_{m_{1}, m_{2}, \ldots m_{n}} c_{1}^{m_{1}} c_{2}^{m_{2}} \ldots c_{n}^{m_{n}}$. Since $\tilde{D}_{i l}(x)$ is also a positive polynomial with constant term $1,\left[\tilde{P}^{(i)}\right]_{l}(x)=\frac{1}{\tilde{D}_{i l}(x)}$ fulfills the requirements to be a proper weighting matrix element.

Therefore, the linear transformation of the variable of the kinetic weighting function influences the kinetic constant parameters of the biochemical reaction rates except for the mass action case, where the denominator terms are constant ones. The transformation, however, does not change the number of kinetics of the complexes.

Lemma 3 Let $P: \mathbb{R}^{n} \rightarrow \mathbb{R}^{\kappa \times m}$ be a kinetic weighting function corresponding to a network of $m$ complexes and $d_{1}, d_{2} \ldots d_{m}$ kinetics $\left(\kappa=\sum_{i=1}^{m} d_{i}\right)$ belonging to the complexes. Let $S$ be a positive diagonal matrix $S=\operatorname{diag}(s)$, where $s \in \mathbb{R}_{+}^{m}$ and let the block-diagonal matrix $H$ constructed as

$$
H=\left(\begin{array}{ccc}
{\left[\operatorname{diag}\left(\frac{1}{s_{1}}\right)\right]_{d_{1}}} & 0_{d_{1} \times d_{2}} & \ldots \\
0_{d_{2} \times d_{1}} & {\left[\operatorname{diag}\left(\frac{1}{s_{2}}\right)\right]_{d_{2}}} & \ldots \\
\vdots & \ddots & \ldots \\
0_{d_{m} \times d_{1}} & \ldots & {\left[\operatorname{diag}\left(\frac{1}{s_{m}}\right)\right]_{d_{m}}}
\end{array}\right)
$$

where $\left[\operatorname{diag}\left(\frac{1}{s_{i}}\right)\right]_{d_{i}}$ denotes a diagonal block of size $d_{i} \times d_{i}$, which contains the constant $\frac{1}{s_{i}}$ in its diagonal and $0_{d_{i} \times d_{j}}$ denotes a block of zeros of size $d_{i} \times d_{j}$. As above, $d_{i}$ is the number of kinetics corresponding to complex $C_{i}$. Then

$$
P(x) S=H^{-1} P(x), \text { i.e. } P(x)=H P(x) S \text { for all } x \in \mathbb{R}_{+}^{n} .
$$

Proof

$$
P(x) S=\left[\begin{array}{cccc}
s_{1} P^{(1)}(x) & 0 & \ldots & 0 \\
0 & s_{2} P^{(2)}(x) & \ldots & 0 \\
& \vdots & & \\
0 & 0 & \ldots & s_{m} P^{(m)}(x)
\end{array}\right]=H^{-1} P(x)
$$

The above lemma technically allows us to 'propagate' a diagonal right multiplier through the kinetic weighting matrix $P(x)$.

The following theorem is the extension for the biochemical reaction networks of the linear conjugacy theorem presented by [21, Theorem 2]. The original statement of the theorem for mass action systems can be found in [20]. 
Theorem 1 Consider a kinetic system of the form (8) with $M=Y \cdot A_{k}$ and $g(x)=P(x) \cdot \Psi(x)$, where $Y \in \mathbb{R}^{n \times m}$ is a complex composition matrix, $A_{k} \in \mathbb{R}^{m \times \kappa}$ is a generalized Kirchhoff matrix, $P(x) \in \mathbb{R}^{\kappa \times m}$ is a kinetic weighting matrix, and $\Psi_{j}(x)=\prod_{i=1}^{n} x_{i}^{Y_{i j}}$ for $j=1, \ldots, m$. Assume that there exists an $n \times n$ positive definite diagonal matrix $T$ such that

$$
Y \cdot A_{b}=T^{-1} \cdot M,
$$

where $A_{b} \in \mathbb{R}^{m \times \kappa}$ is a generalized Kirchhoff matrix. Then the realization $\left(Y, \bar{A}_{k}, \bar{P}\right)$ is linearly conjugate to (8) with

$$
\begin{aligned}
\bar{A}_{k} & =A_{b} \cdot \bar{\Phi}_{T} \\
\bar{P}(x) & =P(T x),
\end{aligned}
$$

and

$$
\bar{\Phi}_{T}=\left(\begin{array}{ccc}
{\left[\operatorname{diag}\left(\Psi_{1}(c)\right)\right]_{d_{1}}} & 0_{d_{1} \times d_{2}} & \ldots \\
0_{d_{2} \times d_{1}} & {\left[\operatorname{diag}\left(\Psi_{2}(c)\right)\right]_{d_{2}}} & \ldots \\
\vdots & \ddots & \ldots \\
0_{d_{m} \times d_{1}} & \ldots & {\left[\operatorname{diag}\left(\Psi_{m}(c)\right)\right]_{d_{m}}}
\end{array}\right) \in \mathbb{R}^{\kappa \times \kappa},
$$

where $\left[\operatorname{diag}\left(\Psi_{i}(c)\right)\right]_{d_{i}}$ denotes a diagonal block of size $d_{i} \times d_{i}$, which contains the constant $\Psi_{i}(c)$ in its diagonal, $0_{d_{i} \times d_{j}}$ denotes a block of zeros of size $d_{i} \times d_{j}$, and $c$ is the vector containing diagonal elements of $T$. As before, $d_{i}$ denotes the number of kinetics corresponding to complex $C_{i}$.

Proof Let us transform the solution of (8) using a positive diagonal transformation $T$ as follows:

$$
\bar{x}=T^{-1} x, \text { i.e. } x=T \bar{x}
$$

Then we can write

$$
\begin{aligned}
\dot{\bar{x}} & =T^{-1} \dot{x}=T^{-1} \cdot M \cdot P(x) \cdot \Psi(x)=T^{-1} \cdot M \cdot P(T \bar{x}) \cdot \Psi(T \bar{x}) \\
& =T^{-1} \cdot M \cdot P(T \bar{x}) \cdot \Phi_{T} \cdot \Psi(\bar{x})=T^{-1} \cdot M \cdot \bar{P}(\bar{x}) \cdot \Phi_{T} \cdot \Psi(\bar{x}),
\end{aligned}
$$

where $\Phi_{T}$ is a diagonal matrix given by

$$
\Phi_{T}=\operatorname{diag}\left(\Psi\left(T \cdot \mathbf{1}^{n}\right)\right)=\operatorname{diag}(\Psi(c)),
$$

and $\mathbf{1}^{n}$ denotes the $n$-dimensional vector with all coordinates equal to one. According to Lemma $2, \bar{P}(\bar{x})$ is a kinetic weighting matrix, and therefore we can apply Lemma 3 as

$$
\bar{P}(\bar{x}) \cdot \Phi_{T}=\bar{\Phi}_{T} \cdot \bar{P}(\bar{x}),
$$

where $\bar{\Phi}_{T}$ is given by Eq. (17). Therefore, using (21) we can write (19) as

$$
\dot{\bar{x}}=T^{-1} \cdot M \cdot \bar{\Phi}_{T} \cdot \bar{P}(\bar{x}) \cdot \Psi(\bar{x}) .
$$

Clearly, if (14) holds, then

$$
\dot{\bar{x}}=Y \cdot A_{b} \cdot \bar{\Phi}_{T} \cdot \bar{P}(\bar{x}) \cdot \Psi(\bar{x})
$$

that is a kinetic system characterized by $\left(Y, \bar{A}_{k}, \bar{P}\right)$, since, according to Lemma 1 , $\bar{A}_{k}$ given in Eq. (15) is a generalized Kirchhoff matrix. 
3.1 Computing linearly conjugate realizations for biochemical systems

In Theorem 1 we assume that the set of complexes and possible kinetics corresponding to the studied kinetic model are known. If the model is given in the ODE form (8) by matrix $M$ and function $g$, one can use the realization algorithm described in [15] which gives a possible realization $\left(Y, A_{k}, P\right)$ of the dynamics. The number of found complexes and the number of kinetics corresponding to each complex determine the size of the blocks of the Kirchhoff matrix (6).

Based on the above, without the loss of generality we can assume that the kinetic model contains $n$ species, $m$ complexes, $d_{i}$ kinetics $(i=1 \ldots m)$ for each complex and let $\sum_{i=1}^{m} d_{i}=\kappa$. Therefore $Y \in \mathbb{R}^{n \times m}$ and $A_{k} \in \mathbb{R}^{m \times \kappa}$, further the $p$-th block of $A_{k}$ (see (6)) is of size $m \times d_{p}$.

In order to find a linearly conjugate realization, according to Theorem 1 , we have to find the generalized Kirchhoff matrix $A_{b} \in \mathbb{R}^{m \times \kappa}$ and a diagonal matrix $T=\operatorname{diag}(c), c \in \mathbb{R}_{+}^{n}$ such that Eq. (14) is fulfilled. We formulate below a linear optimization problem, which constitutes a possible framework for computing linearly conjugate realizations.

It is easy to see that Eq. (14) is a standard linear constraint for the elements of $A_{b}$ and $T^{-1}$, since it can be rewritten as

$$
T^{-1} M-Y A_{b}=0 .
$$

Thus, instead of finding the diagonal matrix $T=\operatorname{diag}(c)$, we are searching for its inverse, which always exists because all the elements of vector $c$ are assumed to be strictly positive. Let us use the following notation

$$
T^{-1}=\tilde{T}=\operatorname{diag}(\tilde{c}) .
$$

Additionally, let us denote the elements of the Kirchhoff matrix $\left[A_{b}\right]_{i j}$ by $a_{i j}$ for $i=1 \ldots m, j=1 \ldots \kappa$. Then Eq. (24) with the positivity constraint on $T$ can be written as

$$
\begin{aligned}
\tilde{c}_{i} & >0 \text { for } i=1 \ldots n \\
\tilde{c}_{i}[M]_{i j}-\sum_{k=1}^{m}[Y]_{i k} a_{k j} & =0 \text { for } i=1 \ldots n, \quad j=1 \ldots \kappa
\end{aligned}
$$

We know that $A_{b}$ is a column conservation matrix. This requirement can be formulated as additional linear equality constraints. For convenience, these constraints are given for each block $(p=1 \ldots m)$ of the Kirchhoff matrix (6) as follows

$$
a_{d_{p} j}=-\sum_{i=1, i \neq d_{p}}^{m} a_{i j} \quad \text { for } j=1 \ldots d_{p}, p=1 \ldots m
$$

Equations (26) and (27) represent $n \times \kappa+\kappa$ equality constrains to find $n+m \times \kappa$ variables.

For practical reasons, it might be required to consider bound constraints on the continuous variables as

$$
\begin{aligned}
a_{i j}^{\mathrm{lb}} \leq a_{i j} \leq a_{i j}^{\mathrm{ub}} & \text { for } i=1 \ldots m, \quad j=1 \ldots \kappa \\
\tilde{c}_{i}^{\mathrm{lb}} \leq \tilde{c}_{i} \leq \tilde{c}_{i}^{\mathrm{ub}} & \text { for } i=1 \ldots n
\end{aligned}
$$


where $a_{i j}^{\mathrm{lb}}, a_{i j}^{\mathrm{ub}}, \tilde{a}_{i j}^{\mathrm{lb}}, \tilde{c}_{i j}^{\mathrm{ub}}$ represent lower and upper bounds for the entries of the Kirchhoff matrix and the scaling parameters, respectively.

Sparse and dense realizations, containing the minimum and maximum number of reactions (directed edges), respectively, can be defined analogously to the mass action case studied in $[31,19]$. To keep track of the presence of individual reactions in the network, binary variables $w \in\{0,1\}^{m \times \kappa}$ can be introduced for each edge as follows: $w_{i j}=1 \Longleftrightarrow a_{i j}>\epsilon$ for $i=1 \ldots m, j=1 \ldots \kappa$, where ' $\Longleftrightarrow$ ' denotes 'if and only if' relationship, and $\epsilon$ is a small positive threshold for distinguishing zero and non-zero rate coefficients. This logical relationship can be formulated in terms of inequality constraints [31] as

$$
\begin{aligned}
& 0 \leq a_{i j}-\epsilon w_{i j} \quad \text { for } i=1 \ldots m, \quad j=1 \ldots \kappa \\
& 0 \leq-a_{i j}+a_{i j}^{\mathrm{ub}} w_{i j} \quad \text { for } i=1 \ldots m, \quad j=1 \ldots \kappa
\end{aligned}
$$

Therefore, the task of finding a sparse network can be given as the following mixed integer linear programming problem:

$$
\underset{w, a, \tilde{c}}{\operatorname{minimize}} \sum_{i=1}^{m} \sum_{j=1}^{\kappa} w_{i j}
$$

subject to the constraints (26), (27), (28), and (29), where the continuous decision variables are $\tilde{c}_{i}$ for $i=1, \ldots, n$, and $a_{k j}$ for $k=1, \ldots, m, j=1, \ldots, \kappa$. The integer (binary) decision variables are $w_{k j}$ for $k=1, \ldots, m$, and $j=1, \ldots, \kappa$. Dense realizations can be determined by modifying the objective function in (30) to maximize the sum of the $w_{i j}$ variables. We note that alternatively, dense realizations can be computed efficiently in polynomial time without integer variables and bound constraints [1]. Additionally, for large enough networks, the sparse realizations can also be determined in polynomial time using standard linear programming [28].

By solving the above outlined optimization problem using an appropriate solver, we obtain the matrix $A_{b}$ and the vector $\tilde{c}$. Then the linearly conjugate network is computed as follows. Inverting the components of the vector $\tilde{c}$ results in $c$ and then $T=\operatorname{diag}(c)$. Finally, the generalized Kirchhoff matrix $\bar{A}_{k}$ and the kinetic weighting matrix $\bar{P}(x)$ of the linearly conjugate network are computed according to Eqs. (15) and (16), respectively, using (17). The above procedures are illustrated by examples in the following section.

\section{Examples}

4.1 Example 1: A kinetic system with limit cycle

First, consider the following dynamical ODE model, which is a modified version of the mass action system corresponding to example A1 in [9]

$$
\begin{aligned}
\frac{d x_{1}}{d t} & =0.05 x_{2}+0.1 x_{1}^{2} x_{2}-\frac{0.2 x_{1}^{2}}{1+2 x_{1}}-\frac{x_{1}}{1+\frac{1}{2} x_{1}} \\
\frac{d x_{2}}{d t} & =1-0.05 x_{2}-0.1 x_{1}^{2} x_{2}+\frac{0.1 x_{1}^{2}}{1+2 x_{1}} .
\end{aligned}
$$



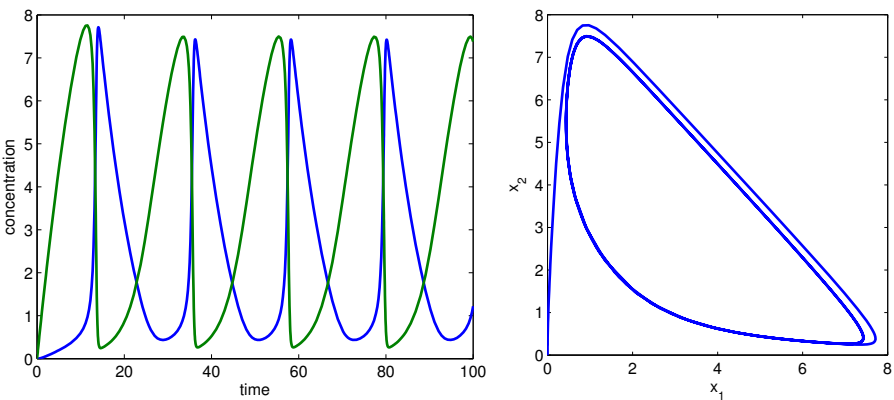

(a) Solution of the dynamic equations

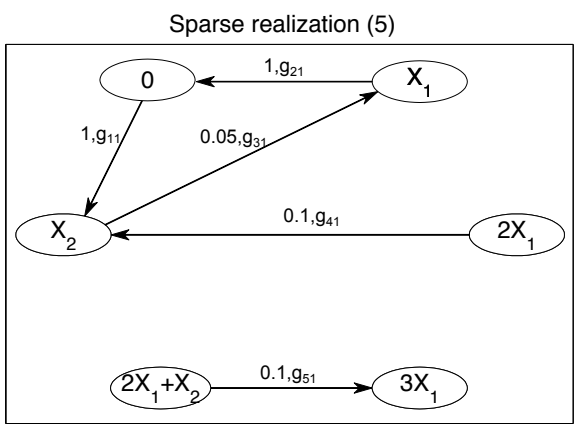

(b) A sparse realization

Dense dynamic equivalent realization(15)

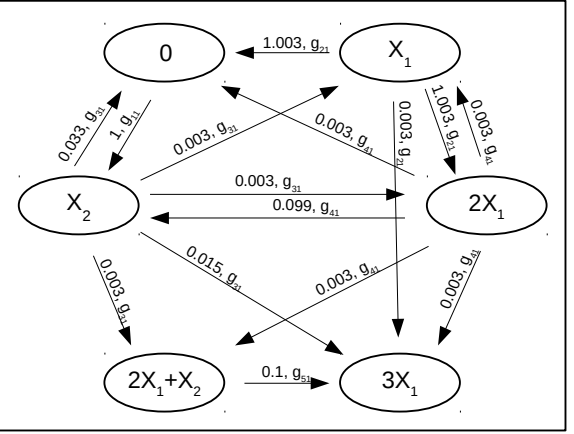

(c) A dense realization

Dense linear conjugated realization(19)

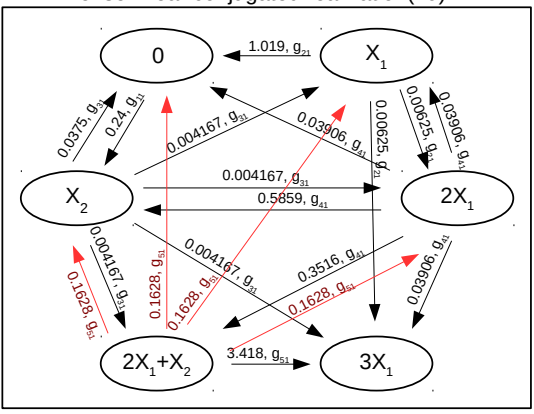

(d) A linearly conjugate dense realization
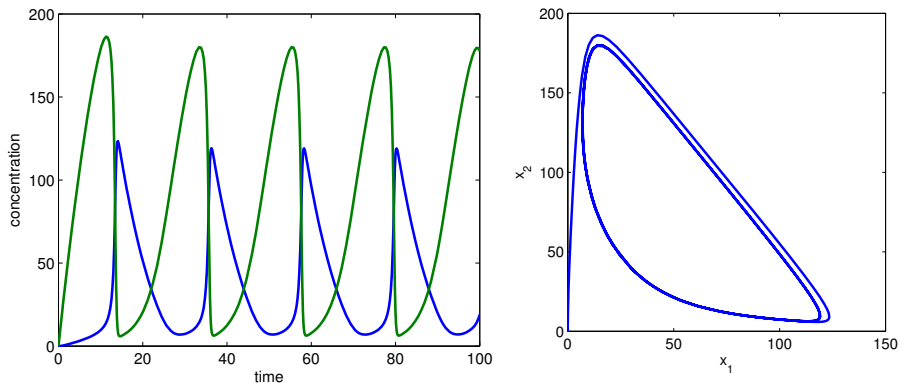

(e) Solution of the linearly conjugate dynamic equations

Fig. 1: Sub-figures 1a-1c contain dynamically equivalent sparse and dense realizations together with the solutions versus time and in the phase space. In sub-figures $1 \mathrm{~d}-1 \mathrm{e}$ a linearly conjugate dense realization is depicted with its solution versus time and also in the phase space. 
The above set of dynamic equations give rise to a stable limit cycle solution, as depicted in Figure 1a for zero initial condition.

A biochemical reaction network realization, which corresponds to the above dynamic equations can be constructed in the following way. First, we assume the complex set $\mathcal{C}=\left\{0, \mathrm{X}_{1}, \mathrm{X}_{2}, 2 \mathrm{X}_{1}, 2 \mathrm{X}_{1}+\mathrm{X}_{2}, 3 \mathrm{X}_{1}\right\}$ as in [9], where 0 denotes the socalled zero complex (see, e.g. [13]). Then, for each complex $C_{1}, \ldots C_{6}$, a kinetic rate function is derived as $g_{11}=1, g_{21}=\frac{x_{1}}{1+x_{1} / 2}, g_{31}=x_{2}, g_{41}=\frac{x_{1}^{2}}{1+2 x_{1}}, g_{51}=x_{1}^{2} x_{2}$ and $g_{61}=x_{1}^{3}$, respectively. This means mass action type rate laws for complexes $C_{1}, C_{3}$ and $C_{5}$, and Michaelis-Menten type reaction kinetics for the complexes $C_{2}$ and $C_{4}$. Then the matrix representation of this bio-CRN realization reads:

$$
\begin{aligned}
& Y=\left(\begin{array}{llllll}
0 & 1 & 0 & 2 & 2 & 3 \\
0 & 0 & 1 & 0 & 1 & 0
\end{array}\right), \\
& A_{k}=\left(\begin{array}{cccccc}
-k_{1} & k_{2} & 0 & 0 & 0 & 0 \\
0 & -k_{2} & k_{3} & 0 & 0 & 0 \\
k_{1} & 0 & -k_{3} & k_{4} & 0 & 0 \\
0 & 0 & 0 & -k_{4} & 0 & 0 \\
0 & 0 & 0 & 0 & -k_{5} & 0 \\
0 & 0 & 0 & 0 & k_{5} & 0
\end{array}\right) \\
& P(x)=\left(\begin{array}{cccccc}
1 & 0 & 0 & 0 & 0 & 0 \\
0 & \frac{1}{1+x_{1} / 2} & 0 & 0 & 0 & 0 \\
0 & 0 & 1 & 0 & 0 & 0 \\
0 & 0 & 0 & \frac{1}{1+2 x_{1}} & 0 & 0 \\
0 & 0 & 0 & 0 & 1 & 0 \\
0 & 0 & 0 & 0 & 0 & 1
\end{array}\right), \quad \Psi(x)=\left(\begin{array}{c}
1 \\
x_{1} \\
x_{2} \\
x_{1}^{2} \\
x_{1}^{2} x_{2} \\
x_{1}^{3}
\end{array}\right)
\end{aligned}
$$

where the parameter values are $k_{1}=1, k_{2}=1, k_{3}=0.05, k_{4}=0.1$ and $k_{5}=0.1$. This is a sparse realization of the dynamical equations with 5 reactions (i.e., it contains the minimum number of reactions), and its reaction graph is shown in Figure 1b.

A dynamically equivalent dense realization of the above can be found with 15 reactions using the optimization framework in [15]. This reaction graph is depicted in Figure 1c, its generalized Kirchhoff matrix is given by

$$
A_{k}^{\text {dense }}=\left(\begin{array}{cccccc}
-1.00 & 1.003 & 0.033 & 10^{-3} & 0 & 0 \\
0 & -1.005 & 10^{-3} & 10^{-3} & 0 & 0 \\
1.00 & 0 & -0.051 & 0.099 & 0 & 0 \\
0 & 10^{-3} & 10^{-3} & -0.103 & 0 & 0 \\
0 & 0 & 10^{-3} & 10^{-3} & -0.10 & 0 \\
0 & 10^{-3} & 0.015 & 10^{-3} & 0.10 & 0
\end{array}\right) .
$$

It was shown in [15] that the dense dynamically equivalent realization has a unique structure and further that, any dynamically equivalent realization contains a subset of the reactions of the dense realization, i.e. the reactions which are not included in the dense reaction cannot appear in any other dynamically equivalent realization.

A linearly conjugate realization Now we compute a dense linearly conjugate realization of the dynamic system (31). First, we solve the optimization problem described in subsection 3.1 with the following tuning parameters:

- threshold value $\epsilon=10^{-6}$ 
- bound constraints for $A_{b}: a_{i j}^{\mathrm{lb}}=0$ and $a_{i j}^{\mathrm{ub}}=100$ for $i=1 \ldots m$ and $j=1 \ldots \kappa$,

- bound constraints for $\tilde{c}$ : $\tilde{c}_{i}^{\mathrm{lb}}=0.01$ and $\tilde{c}_{i}^{\mathrm{ub}}=1000$ for $i=1 \ldots m$ and $j=$ $1 \ldots \kappa$.

We used GLPK (GNU Linear Programming Kit) through the YALMIP interface [24] in MATLAB 2012b.

The solution of the optimization resulted in the following Kirchhoff and scaling matrices

$$
\begin{aligned}
A_{b} & =\left(\begin{array}{cccccc}
-0.24 & 0.163 & 9.0 \cdot 10^{-3} & 10^{-3} & 10^{-3} & 0 \\
0 & -0.165 & 10^{-3} & 10^{-3} & 10^{-3} & 0 \\
0.24 & 0 & -0.013 & 0.015 & 10^{-3} & 0 \\
0 & 10^{-3} & 10^{-3} & -0.027 & 10^{-3} & 0 \\
0 & 0 & 10^{-3} & 9.000 \cdot 10^{-3} & -0.025 & 0 \\
0 & 10^{-3} & 10^{-3} & 10^{-3} & 0.021 & 0
\end{array}\right) \\
T(x)^{-1} & =\left(\begin{array}{cc}
0.16 & 0 \\
0 & 0.24
\end{array}\right) .
\end{aligned}
$$

Then, following the procedure described in subsection 3.1, first we obtain $T$ and matrix $\bar{\Phi}_{T}$ by (17), as

$$
\begin{aligned}
T & =\left(\begin{array}{cc}
6.25 & 0 \\
0 & 4.1667
\end{array}\right), \\
\bar{\Phi}_{T} & =\operatorname{diag}\left(\left[1,0.16,0.24,0.0256,6.144 \cdot 10^{-3}, 4.096 \cdot 10^{-3}\right]^{T}\right) .
\end{aligned}
$$

Finally, Equations (15) and (16) give the dense generalized Kirchhoff matrix of the linearly conjugate network

$$
\bar{A}_{k}^{\text {dense }}=\left(\begin{array}{cccccc}
-0.2400 & 1.019 & 0.03750 & 0.03906 & 0.1628 & 0 \\
0 & -1.031 & 4.167 \cdot 10^{-3} & 0.03906 & 0.1628 & 0 \\
0.2400 & 0 & -0.05417 & 0.5859 & 0.1628 & 0 \\
0 & 6.250 \cdot 10^{-3} & 4.167 \cdot 10^{-3} & -1.055 & 0.1628 & 0 \\
0 & 0 & 4.167 \cdot 10^{-3} & 0.3516 & -4.069 & 0 \\
0 & 6.250 \cdot 10^{-3} & 4.167 \cdot 10^{-3} & 0.03906 & 3.418 & 0
\end{array}\right),
$$

and in its transformed kinetic weighting function

$$
\bar{P}(x)=\left(\begin{array}{cccccc}
1 & 0 & 0 & 0 & 0 & 0 \\
0 & \frac{1}{1+6.25 \cdot 0.5 x_{1}} & 0 & 0 & 0 & 0 \\
0 & 0 & 1 & 0 & 0 & 0 \\
0 & 0 & 0 & \frac{1}{1+6.25 \cdot 2 x_{1}} & 0 & 0 \\
0 & 0 & 0 & 0 & 1 & 0 \\
0 & 0 & 0 & 0 & 0 & 1
\end{array}\right) .
$$

The reaction graph of the resulted linearly conjugate network $\left(Y, \bar{A}_{k}^{\text {dense }}, \bar{P}\right)$ is depicted in Figure 1d. It can be seen that there are 4 edges, i.e. 4 reactions, highlighted in red color, which do not appear in the dense dynamically equivalent realization (see Figure 1c).

For the comparison of the dynamics of the original and the linearly conjugate network, the solutions corresponding to the zero initial condition is depicted in the time domain and in the phase space, see Figure 1e, where we can see the effect if scaling and the qualitative similarities with the original solutions in Figure 1a. 
4.2 Example 2: searching for a reversible linearly conjugate realization

Johnston and Siegel considered the following case study as Example 1 in [20]:

$$
\begin{aligned}
\frac{d x_{1}}{d t} & =2 k_{3} x_{1} x_{2}-2 k_{4} x_{1}^{2} \\
\frac{d x_{2}}{d t} & =k_{4} x_{1}^{2}-2 k_{2} x_{1} x_{2}^{3}+k_{1} x_{1} x_{2}^{2}-k_{3} x_{1} x_{2} .
\end{aligned}
$$

with the complex set $\left\{C_{1}=\mathrm{X}_{1}+2 \mathrm{X}_{2}, C_{2}=\mathrm{X}_{1}+3 \mathrm{X}_{2}, C_{3}=\mathrm{X}_{1}+\mathrm{X}_{2}, C_{4}=\right.$ $\left.3 \mathrm{X}_{1}, C_{5}=2 \mathrm{X}_{1}, C_{6}=\mathrm{X}_{2}\right\}$. They showed that this dynamical system does not have a weakly reversible realization, but has a linearly conjugate reversible realization with zero deficiency.

Let us consider the modified dynamical equations with some non mass action reaction rates and fixed constants $\left(k_{i}=1\right.$ for $\left.i=1 \ldots 4\right)$ as follows:

$$
\begin{aligned}
\frac{d x_{1}}{d t} & =2 x_{1} x_{2}-\frac{2 x_{1}^{2}}{1+0.5 x_{1}^{2}} \\
\frac{d x_{2}}{d t} & =\frac{x_{1}^{2}}{1+0.5 x_{1}^{2}}-2 x_{1} x_{2}^{3}-x_{1} x_{2}+\frac{x_{1} x_{2}^{2}}{1+x_{1} x_{2}^{2}} .
\end{aligned}
$$

Then the above kinetic system has the following sparse realization with 4 reactions

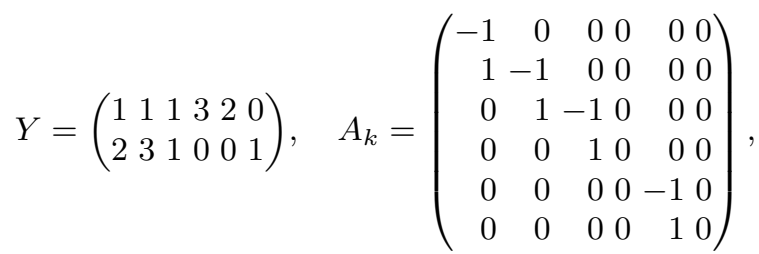

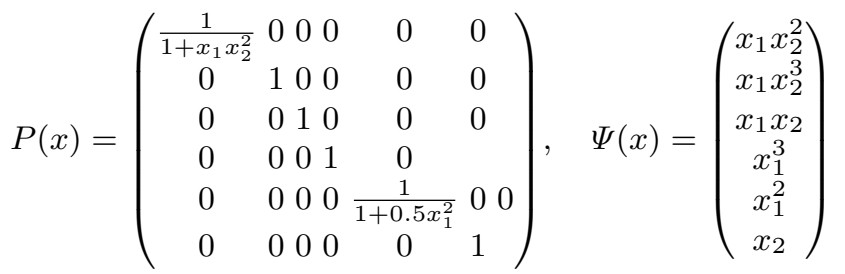

We are also interested in finding weakly reversible realization for the system. A similar analysis that was derived in [20] shows that this network does not have a weakly reversible dynamically equivalent realization with the given complex set.

A linearly conjugate realization However, there exists a linearly conjugate reversible realization with the following matrices

$$
\begin{aligned}
\bar{A}_{k} & =\left(\begin{array}{cccccc}
-2.0 & 4.0 & 0 & 0 & 0 & 0 \\
2.0 & -4.0 & 0 & 0 & 0 & 0 \\
0 & 0 & -2.0 & 0 & 4.0 & 0 \\
0 & 0 & 0 & 0 & 0 & 0 \\
0 & 0 & 2.0 & 0 & -4.0 & 0 \\
0 & 0 & 0 & 0 & 0 & 0
\end{array}\right) \\
T^{-1} & =\left(\begin{array}{cc}
0.5 & 0 \\
0 & 1.0
\end{array}\right)
\end{aligned}
$$




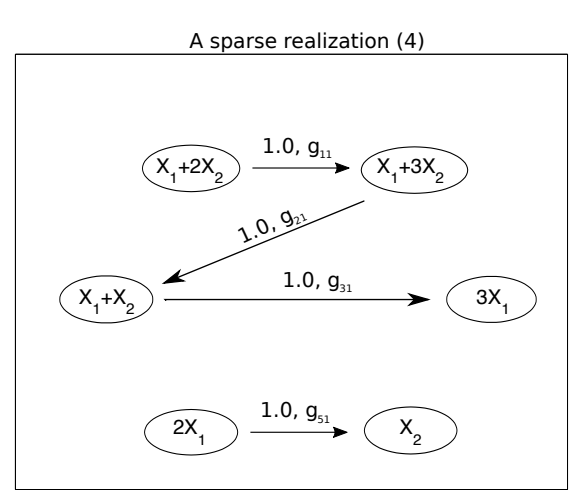

(a) A sparse realization

Reverzible linearly conjugated realization (4)

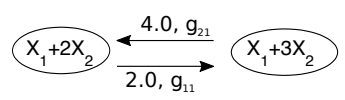
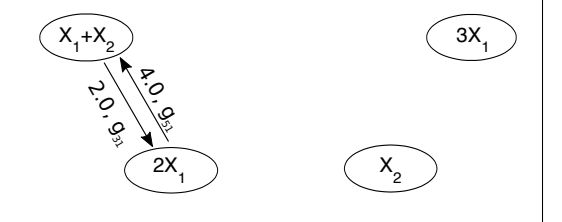

(c) A linearly conjugate reversible realization

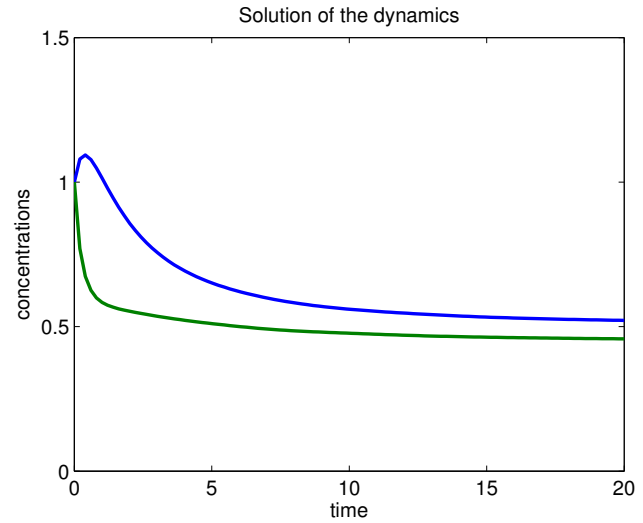

(b) Solution of the dynamic equations

Solution of the dynamics

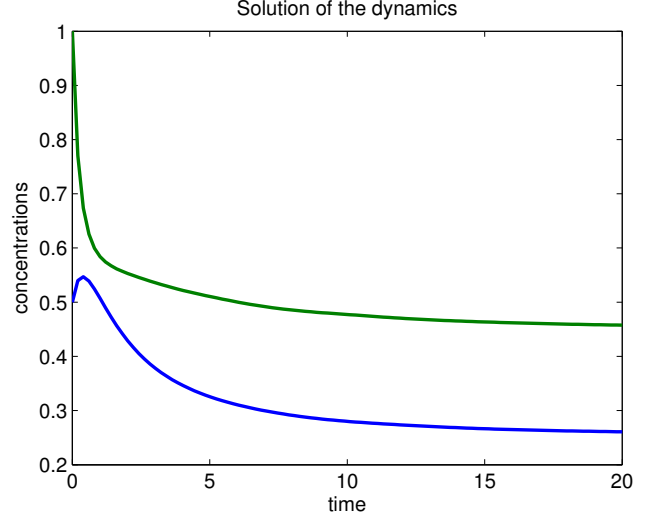

(d) Solution of the linearly conjugate dynamics

Fig. 2: Illustration of Example 2. The reaction graphs for a sparse realization of the dynamic equations and a linearly conjugate reversible realization are depicted in $2 \mathrm{a}$ and $2 \mathrm{c}$. The solutions of the corresponding equations are shown in figures $2 \mathrm{~b}$ and $2 \mathrm{~d}$, respectively.

The reaction graphs of the original and the linearly conjugate system and the corresponding trajectories are depicted in Figure 2.

\section{Summary}

Linear conjugacy was introduced in this paper for a wide class of kinetic models containing rational terms in the reaction rates, which is a common property of biochemical models. The reaction graph of these models allow multiple directed edges corresponding to different types of kinetics. It is assumed that the set of complexes and the set of possible kinetics corresponding to source complexes are either known or determined by the realization algorithm [15], and then these two sets are fixed during the computation. 
It was shown that the generalized Kirchhoff matrix and the rate weighting matrix of the linearly conjugate network can be computed using the parameters of the applied diagonal transformation. This fact allows us to apply efficient computation methods to find linearly conjugate realizations corresponding to the ODEs of a given kinetic system.

Two illustrative examples show that similarly to the mass action case, the set of possible linearly conjugate realizations may contain more reaction graph structures than the set of dynamically equivalent realizations. Furthermore, it was also shown that the applied matrix-based model structure is suitable for the optimization-based search of linearly conjugate reaction networks having preferred properties like density/sparsity or reversibility.

Acknowledgements AG is supported by the funding from EU FP7 ITN "NICHE", project no. 289384. KMH acknowledges the funding from the National Research, Development and Innovation Office - NKFIH through grant No. 115694. GSz acknowledges the support of the National Research, Development and Innovation Office - NKFIH through grant No. NF104706. The authors thank Bernadett Ács for carefully reading the manuscript.

\section{References}

1. B. Ács, G. Szederkényi, Zs. Tuza, and Z. A. Tuza. Computing linearly conjugate weakly reversible kinetic structures using optimization and graph theory. MATCH Communications in Mathematical and in Computer Chemistry, 74(3):481-504, 2015.

2. D. F. Anderson. A proof of the global attractor conjecture in the single linkage class case. SIAM Journal on Applied Mathematics, 71:1487-1508, 2011.

3. D. Angeli, P. De Leenher, and E. D. Sontag. A Petri net approach to the study of persistence in chemical reaction networks. Mathematical Biosciences, 210:598-618, 2007.

4. M. Banaji and C. Pantea. Some results on injectivity and multistationarity in chemical reaction networks. arXiv:1309.6771 [math.DS], 2015.

5. V. Chellaboina. Modeling and analysis of mass-action kinetics. IEEE Control Systems Magazine, pages 60-78, 2009.

6. G. Craciun. Toric differential inclusions and a proof of the global attractor conjecture. arXiv:1501.02860v2 [math.DS], 2016.

7. G. Craciun and C. Pantea. Identifiability of chemical reaction networks. Journal of Mathematical Chemistry, 44:244-259, 2008.

8. G. Craciun and M. Feinberg. Multiple equilibria in complex chemical reaction networks: I. The injectivity property. SIAM Journal on Applied Mathematics, 65(5):1526-1546, January 2005.

9. A. Császár, L. Jicsinszky, and T. Turányi. Generation of model reactions leading to limit cycle behaviour. React. Kinet. Catal. Lett, 18(1-2):65-71, 1981.

10. P. Donnell and M. Banaji. Local and global stability of equilibria for a class of chemical reaction networks. SIAM Journal on Applied Dynamical Systems, 12:899-920, 2013.

11. P. Érdi and J. Tóth. Mathematical Models of Chemical Reactions. Theory and Applications of Deterministic and Stochastic Models. Manchester University Press, Princeton University Press, Manchester, Princeton, 1989.

12. G. Farkas. Kinetic lumping schemes. Chemical Engineering Science, 54(June 1998):39093915, 1999.

13. M. Feinberg. Chemical reaction network structure and the stability of complex isothermal reactors - I. The deficiency zero and deficiency one theorems. Chemical Engineering Science, 42(10):2229-2268, 1987.

14. M. Feinberg. Necessary and sufficient conditions for detailed balancing mass action systems of arbitrary complexity. Chemical Engineering Science, 44(9):1819-1827, January 1989.

15. A. Gábor, K. M. Hangos, J. R. Banga, and G. Szederkényi. Reaction network realizations of rational biochemical systems and their structural properties. Journal of Mathematical Chemistry, 53:1657-1686, 2015. 
16. V. Hárs and J. Tóth. On the inverse problem of reaction kinetics. In M Farkas and L Hatvani, editors, Qualitative Theory of Differential Equations, volume 30 of Coll. Math. Soc. J. Bolyai, pages 363-379. North-Holland, Amsterdam, 1981.

17. B. Hernandez-Bermejo, V. Fairen, and L. Brenig. Algebraic recasting of nonlinear ODEs into universal formats. J. Phys. A, Math. Gen., 31:2415-2430, 1998.

18. F. Horn and R. Jackson. General mass action kinetics. Archive for Rational Mechanics and Analysis, 47(2):81-116, 1972.

19. M. D. Johnston, D. Siegel, and G. Szederkényi. A linear programming approach to weak reversibility and linear conjugacy of chemical reaction networks. Journal of Mathematical Chemistry, 50:274-288, 2012.

20. M. D. Johnston and D. Siegel. Linear conjugacy of chemical reaction networks. Journal of Mathematical Chemistry, 49:7(7):1263-1282, April 2011.

21. M. D. Johnston, D. Siegel, and G. Szederkényi. Dynamical equivalence and linear conjugacy of chemical reaction networks: new results and methods. MATCH Commun. Math. Comput. Chem., 68:443-468, 2012.

22. M. D. Johnston, D. Siegel, and G. Szederkényi. Computing weakly reversible linearly conjugate chemical reaction networks with minimal deficiency. Mathematical Biosciences, 241:1(1):88-98, January 2013.

23. G. Lipták, G. Szederkényi, and K. M. Hangos. Computing zero deficiency realizations of kinetic systems. Systems \& Control Letters, 81:24-30, 2015.

24. J. Löfberg. YALMIP : A Toolbox for Modeling and Optimization in MATLAB. In Proceedings of the CACSD Conference, Taipei, Taiwan, 2004.

25. M. Mincheva and D. Siegel. Stability of mass action reaction-diffusion systems. Nonlinear Analysis: Theory, Methods $\mathcal{E}$ Applications 56(8):1105-1131, 2004

26. M. Mincheva and M. R. Roussel. Graph-theoretic methods for the analysis of chemical and biochemical networks. I. Multistability and oscillations in ordinary differential equation models. Journal of mathematical biology. 55(1):61-86, 2007.

27. S. Müller and G. Regensburger. Generalized mass action systems: Complex balancing equilibria and sign vectors of the stoichiometric and kinetic-order subspaces. SIAM Journal on Applied Mathematics, 72:1926-1947, 2012.

28. J. Rudan, G. Szederkényi, and K. M. Hangos. Efficient computation of alternative structures for large kinetic systems using linear programming. MATCH Commun. Math. Comput. Chem., 71:71-92, 2014.

29. N. Samardzija, L. D. Greller, and E. Wassermann. Nonlinear chemical kinetic schemes derived from mechanical and electrical dynamical systems. Journal of Chemical Physics, 90 (4):2296-2304, 1989.

30. G. Shinar and M. Feinberg. Structural sources of robustness in biochemical reaction networks. Science, 327(5971):1389-1391, 2010.

31. G. Szederkényi. Computing sparse and dense realizations of reaction kinetic systems. Journal of Mathematical Chemistry, 47(2):551-568, 2010.

32. G. Szederkényi, K. M. Hangos, and Zs. Tuza. Finding weakly reversible realizations of chemical reaction networks using optimization. MATCH Communications in Mathematical and in Computer Chemistry, 67:193-212, 2012.

33. L. Szili and J. Tóth. On the origin of Turing instability. Journal of Mathematical Chemistry 22(1):39-53, 1997.

34. A. I. Vol'pert. Differential equations on graphs. Mathematics of the USSR-Sbornik, 17(4):571-582, 1972. 